\title{
Metastable Hadronic States in Helium
}

\author{
Dezso Horváth of the Research Institute for Particle and Nuclear Physics, \\ Budapest, reports that a recent workshop highlighted the questions which need \\ to be addressed by theoreticians in understanding why a significant fraction of \\ antiprotons trapped in helium live for a relatively long time.
}

Exotic atoms are undergoing an exciting revival these days. The last time this happened was in the late-1970s when a sharp temperature dependence (temperature resonance) was found by a group at the Joint Institute for Nuclear Research, Dubna, in the formation of muonic molecules of hydrogen isotopes. Muon-catalysed fusion suddenly became very interesting, and measurements started wherever low-energy muons were available.

This time it is the longevity of the antiprotonic helium atom (a so-called "atomcule" comprising an antiproton, an electron and a $\mathrm{He}^{++} \alpha$-particle [1]) which is causing all the excitement. More and more theorists have been attracted to the field after CERN's PS205 collaboration (Tokyo, Okazaki, Munich, CERN, and Budapest) observed a laser-induced resonant de-excitation [2] of this metastable state in 1993. Although calculations have only been made for the $\overline{\mathrm{p}}-\alpha-\mathrm{e}^{-}$three-body system following the ideas of Condo and Russell, several other approaches have been put forward to explain the anomalously long lifetime of antiprotons in helium. Some 3\% of all antiprotons stopped in helium lives for about $3 \mathrm{~ms}$ while the remainder annihilates typically in $10^{-12} \mathrm{~s}$. A second resonance found last year [3] confirmed the atomic model as the only possible interpretation.

In addition to measuring the wavelength of the metastable transition, the antiproton population of the long-living states has also been studied by resonance de-excitation of the $\overline{\mathrm{p}}-\alpha-\mathrm{e}^{-}$state using two consecutive laser pulses with different timings [4]. These high precision measurements have challenged the theoreticians to start making three-body Coulomb calculations. Unfortunately, there is only one source of low-energy antiprotons available, namely the Low Energy Antiproton Ring (LEAR) at CERN, so interested experimentalists are obliged to make their experiments there (and even LEAR is scheduled to be shut down owing to financial cutbacks).

A symposium in March 1994 in Erice on the physics of exotic atoms and molecules organized by E. Zavattini and C. Rizzo, members of CERN'S OBELIX collaboration (Bologna, Brescia, Cagliari, Dubna, Frascati, Legnaro, Padova, Pavia, Torino, Trieste, and Udine), demonstrated so much

Dezso Horváth (horvath@rmki.kfki.hu) is an ex perimental physicist with the Research Institute for Particle and Nuclear Physics, Hungarian Academy of Sciences. interest in the long-living antiprotonic helium that J. Révai and myself decided to arrange a small informal workshop in early 1995 , when the results of the autumn 1994 runs of PS-205 and OBELIX would be available. The Workshop on Metastable Hadronic States in Helium (26-28 January 1995; Balatonfüred) included a review by $\mathrm{T}$. Yamazaki (INS, Tokyo), the spokesman and the main mover of the PS205 collaboration, as well as talks alternating between theoretical and experimental aspects. J. Russell (Cincinnati) in summarizing the workshop suggested that the principle questions to be answered by theoreticians are:

- Why do we only see a $3 \%$ metastable fraction when theoretical calculations for the initial population of the potentially metastable antiprotonic orbits give values as high as $20-30 \%$ ? G. Korenman of Moscow explained this discrepancy in terms of collisional quenching of those states where the antiprotonic orbit is large enough to stick out from behind the Pauli shield provided by the electron. He predicted a sharp increase of metastable populations at extreme low densities (below $0.1 \mathrm{mbar}$ ), thus giving impetus to the OBELIX collaboration to observe the trapping effect at low pressures.

- What causes the large isotope effects observed between ${ }^{4} \mathrm{He}$ and $3 \mathrm{He}$ (different metastable lifetimes; appearance of a shorter lived but still metastable fraction)? - What is the ultimate observable metastable state in $3 \mathrm{He}$ ? It is very important to answer this question when searching for resonances in ${ }^{3} \mathrm{He}$.

- What is the mechanism of quenching the metastable states by certain impurities (e.g., hydrogen) given the fact that collisions with other noble gas atoms (or with helium itself) do not affect the metastable atom very much?

$J$. Eades (CERN), in reviewing experimental prospects, emphasized that in addition to laser resonance studies (searching for other transitions; checking impurity effects on the transition wavelengths and populations; measuring the resonance shapes at improved resolution), PS205 plans to analyze fluorescence photons in the visible from antiproton transitions in the metastable region when as many as $10^{9}$ antiprotons are stopped in the helium target during a 100-200 ns pulse from LEAR. Meanwhile, OBELIX will study $\overline{\mathrm{p}}-\alpha-\mathrm{e}^{-}$ formation at pressures around $1 \mathrm{mbar}$, and a recently approved LEAR experiment is about to study antiprotonic helium and hydrogen at $10^{-3}$ mbar using a Penning trap device [5].

If LEAR survives after 1996, there are many interesting measurements to be made [6], including:

- Studies of the hyperfine structure from the interaction between the antiproton orbit and the electron spin in $\overline{\mathrm{p}}-\alpha-\mathrm{e}$ : As shown experimentally by T. Yamazaki and supported by precise theoretical calculations, this should allow the determination of the magnetic moment of the antiproton to a precision of $10^{-7}$, some five orders of magnitude better than today's value.

- Precise studies of antiprotonic states. It should be possible to extract bunches of $10^{6}-10^{7}$ cooled antiprotons from a Penning trap, accelerate them to various energies (around tens of $\mathrm{eV}$ ), and let them interact with a helium jet target. The antiprotons will have different and well-controlled incident energies when captured by helium atoms, and will populate states with different angular momentum distributions thus allowing the populations to be investigated.

- High-precision spectroscopy of an atomic $\overline{\mathrm{p}}-\alpha-\mathrm{e}^{-}$beam using a beam generated from the Penning trap device. This is feasible owing to the very narrow opening angle for $\overline{\mathrm{p}}-\alpha$-e $\mathrm{e}^{-}$production [5] and because the metastable atoms tend to live for a long time when isolated.

[1] Iwasaki M. et al., Phys. Rev. Lett. 67 (1991) 1246; Yamazaki T. et al., Nature 361 (1993) 238; Eades J., Europhys. News 24 (1993) 172. [2] Morita N. et al., Phys. Rev. Lett. 72 (1994) 1180.

[3] Maas F.E. et al., Phys. Rev. Lett. (to be published).

[4] Hayano R.S. et al., Phys. Rev. Lett. 73 (1994) 1485; Erratum, Phys. Rev. Lett. 73 (1994) 3181

[5] PS200 Collaboration, CERN SPSLC 95-2, SPSLC/P-285 (1995).

6] PS205 Collaboration, CERN SPSLC 95-12, SPSLC/I-201 (1995).

The Workshop on Metastable Hadronic States in Helium (26-28 January 1995; Balatonfüred, Hungary) was attended by over 40 participants. Generous support from the Hungarian Academy of Sciences' International Workshops on Theoretical Physics programme covered the expenses of 14 junior and senior scientists from east and central Europe who would otherwise have been unable to attend. Further workshops in the series are planned for 1996 (Udine, Italy) and 1997 (Hungary). 